\title{
Riesgos Psicosociales en el Equipo de Salud de Hospitales Públicos de la Provincia de Córdoba, Argentina
}

\section{PSYCHOSOCIAL RISKS IN THE HEALTH TEAM PUBLIC HOSPITALS IN THE PROVINCE OF CÓRDOBA, ARGENTINA}

\author{
Gabriel Esteban Acevedo', Julieta Sánchez², María Alejandra Farías³, Alicia Ruth Fernández ${ }^{4}$ \\ 1. Profesor Titular en Medicina Preventiva y Social, Doctor en Medicina y Cirugia, Médico Especialista en Salud Pública. Director del CeGeSST de la Escuela de \\ Salud Pública. \\ 2. Profesora Asistente en Medicina Preventiva y Social, Magister en Salud Pública, Odontóloga. Investigadora del CeGeSST de la Escuela de Salud Pública. \\ 3. Profesora Asistente en Medicina Preventiva y Social, Magíster en Salud Pública, Bioquímica. Investigadora del CeGeSST de la Escuela de Salud Pública. \\ 4. Profesora Titular de la Escuela de Salud Pública. Doctora en Medicina y Cirugía, Médica.
}

\section{RESUMEN}

El objetivo de esta investigación fue determinar el nivel de riesgo psicosocial en el trabajo de los integrantes de los equipos de salud de hospitales dependientes del Ministerio de Salud de la Provincia de Córdoba. Se diseñó un estudio descriptivo, de corte transversal, sobre 844 trabajadores del equipo de salud pertenecientes a 23 hospitales públicos, seleccionados mediante muestreo probabilístico aleatorio simple, con un nivel de confianza del 95\% y un error muestral del 5\%. Se aplicó el instrumento SUSESO ISTAS-21, versión corta de la Superintendencia de Seguridad Social (SUSESO) del Gobierno de Chile.

Se determinó que los riesgos psicosociales en situación más desfavorables (riesgo Alto) se correspondieron con las dimensiones: Exigencias psicológicas (57,7\%), Apoyo social y calidad de liderazgo $(56,2 \%)$ y Doble presencia (64\%). El riesgo Medio predominó en la dimensión Compensaciones (39\%) y el riesgo Bajo resultó más frecuente en la dimensión Trabajo activo y desarrollo de habilidades (42,9\%).

Se concluye que este personal sanitario presenta una elevada frecuencia de exposición a riesgos psicosociales en el ámbito laboral, lo que genera la necesidad de indagar sobre los factores condicionantes de estos riesgos y establecer acciones de promoción de ambientes de trabajo más saludables.

(Acevedo G, Sánchez J, Farías M, Fernández A, 2013. Riesgos Psicosociales en el Equipo de Salud de Hospitales Públicos de la Provincia de Córdoba, Argentina. Cienc Trab. Sep-Dic; 15 [48]: 140-147).

Palabras claves: TRABAJADORES DE SALUD, RIESGOS LABORALES, AGOTAMIENTO PROFESIONAL, ESTRÉS PSICOLÓGICO, AMBIENTE DE TRABAJO.

\section{INTRODUCCIÓN}

El escenario de salud de las últimas décadas se ha caracterizado por una dinámica de constante cambio, motivado principalmente por importantes modificaciones en el perfil de la población

Correspondencia / Correspondence:

Gabriel Esteban Acevedo

Escuela de Salud Pública, Universidad Nacional de Córdoba.

Enrique Barros esquina Enfermera Gordillo

Ciudad Universitaria. CP: 5000

e-mail: geacevedo_esp@fcm.unc.edu.ar

Tel.: 543514334042 - Fax: 4333023

Recibido: 20 de Noviembre 2013 / Aceptado: 08 Diciembre 2013

\section{ABSTRACT}

The objective of this research was to determine the level of psychosocial risk in the work of the members of health teams hospitals of the Ministry of Health of the Province of Córdoba.

It was designed a descriptive cross-sectional study, about 844 workers of the health team from 23 public hospitals, selected using simple random probability sampling, with a confidence level of 95\% and a sampling error of 5\%. The instrument SUSESO ISTS-21, short version of the Superintendence of Social Security (SUSESO, in Spanish acronym) of the Chilean Government was applied.

It was determined that psychosocial risks within more unfavorable situation (High risk) were matched with dimensions: Psychological Requirements (57,7\%), social support and quality of leadership (56.2\%) and Double presence (64\%). The middle risk dominated the Compensation dimension (39\%) and Low risk was more frequent in the active job and skill development dimension (42,9\%).

We conclude that the medical staff has a high frequency of exposure to psychosocial risks in the workplace, creating the need to investigate the determinants of these risks and establish measures to promote healthier work environments.

Key words: HEALTH WORKERS, OCCUPATIONAL HAZARDS, BURNOUT, PSYCHOLOGICAL STRESS, WORK ENVIRONMENT (sociales, demográficas y epidemiológicas) y por la vertiginosa innovación tecnológica en los servicios. ${ }^{1}$ Estos cambios y sus implicancias en los sistemas sanitarios generan nuevos desafíos y exigencias para los trabajadores del sector dado que provocan: mayor complejidad de las tareas, necesidades de actualización de competencias, incremento en la demanda de asistencia, nuevas formas de organización de los procesos asistenciales, prolongación de jornadas de trabajo, inestabilidad del empleo, afectación de las relaciones sociales y familiares, y también una modificación en el perfil de salud de los propios trabajadores, donde asumen especial relevancia los problemas de origen psicosocial.

En este contexto se observa un interés creciente en la investigación de los factores psicosociales, que son condiciones presentes en situaciones laborales relacionadas con la organización del trabajo, el tipo de puesto, la realización de la tarea, e incluso con el entorno, que afectan al desarrollo del trabajo y a la salud de las 
personas trabajadoras. ${ }^{2}$ Los factores psicosociales pueden favorecer o perjudicar la actividad laboral y la calidad de vida laboral de las personas. En el primer caso fomentan el desarrollo personal de los individuos, mientras que cuando son desfavorables perjudican su salud y su bienestar, configurando un riesgo psicosocial que es fuente de estrés laboral, o estresor, y que tiene el potencial de causar daño psicológico, físico, o social a los individuos. ${ }^{1}$ Se ha postulado que el trabajador, dependiendo de sus características individuales (tipo de personalidad, educación, edad, experiencia, grado de motivación, etc.) va a percibir y asumir los efectos de los riesgos psicosociales de diferentes maneras, por lo que la exposición a este tipo de riesgos no deteriora necesariamente la salud del trabajador, aunque, y debido a que se ha demostrado que los riesgos psicosociales en el trabajo suelen tener carácter crónico, la exposición a los mismos supone una tensión continua, que se manifiesta a través de alteraciones fisiológicas y psicológicas, conocidas comúnmente como estrés laboral, que conduce a un deterioro de la salud de las personas durante el desempeño de su trabajo e incluso fuera de él. ${ }^{4}$

Investigaciones sobre esta problemática, llevadas a cabo tanto sobre trabajadores de diferentes tipos de actividades como sobre el personal sanitario en particular, revelan que el estrés laboral, mantenido en el tiempo, puede constituir un factor de riesgo de enfermedad cardiovascular, afecciones músculo-esqueléticas, depresión; abuso de sustancias, trastornos psiquiátricos, Burnout y baja autopercepción de la salud. ${ }^{5-7,8,9}$

Además de los efectos sobre la salud del trabajador, la exposición a riesgos psicosociales puede provocar consecuencias negativas para la organización en la que trabaja, relacionándose con reducción de la satisfacción laboral, aumento del ausentismo, mayor accidentabilidad e incremento de la propensión a abandonar la organización y, como consecuencia de estos, afectar la calidad de la atención que el personal de salud brinda a la población que asiste. ${ }^{10-11}$

Existen estudios en diversos países donde se ha investigado sobre la presencia de los riesgos psicosociales en los servicios de salud, tales como la cuarta Encuesta de Condiciones de Trabajo en España elaborada por el Instituto Nacional de Seguridad e Higiene en el Trabajo, a partir de la que se determinó que los riesgos psicosociales en el trabajo fueron una de las principales causas de enfermedades y de accidentes laborales, donde el 74,9\% del personal sanitario encuestado señaló este tipo de riesgos relacionados a los accidentes laborales. $^{12}$

No obstante, en Argentina, como en otros países de Sudamérica, se observa una carencia de este tipo de estudios, motivo por el cual se desarrolló esta investigación que tuvo como objetivo determinar el nivel de riesgo psicosocial en el trabajo de los integrantes de los equipos de salud de hospitales dependientes del Ministerio de Salud de la Provincia de Córdoba.

\section{MATERIAL Y MÉTODOS}

\section{Diseño y muestra}

Se diseñó un estudio descriptivo, de corte transversal, en el que se aplicó un cuestionario autoadministrado, durante el último trimestre del año 2011, sobre 844 trabajadores del equipo de salud de 23 hospitales públicos (9 de Córdoba Capital y 18 del interior provincial) dependientes del Ministerio de Salud de la Provincia de Córdoba, Argentina, que incluye a: médicos, enfermeros (profesionales y auxiliares), bioquímicos, psicólogos, odontólogos, farmacéuticos, licenciados en Kinesiología y Fisioterapia, licenciados en Fonoaudiología, licenciados en Trabajo Social, técnicos universitarios en Radiología, Laboratorio y Hemoterapia, que en su conjunto ascendían a 8.371 trabajadores, según datos del Ministerio de Salud de la Provincia de Córdoba.

Para la selección de los encuestados se aplicó un muestreo probabilístico aleatorio simple, con un nivel de confianza del 95\% y un error muestral del 5\%. Las personas seleccionadas fueron contactadas en su lugar de trabajo e invitadas a responder el cuestionario; se distribuyeron 1.200 cuestionarios con una tasa de respuesta del 70,33\%.

\section{Instrumento de recolección de datos}

Se empleó el instrumento SUSESO ISTAS-21, versión corta, validado, estandarizado y adaptado por investigadores chilenos a requerimiento de la Superintendencia de Seguridad Social (SUSESO) del Gobierno de Chile. ${ }^{13}$ Este instrumento, que permite la evaluación de riesgos psicosociales en el trabajo, consta de 5 dimensiones y 20 subdimensiones, y en cuyo cuestionario se incluye una pregunta por cada sub-dimensión, con cinco opciones de respuesta: siempre; la mayoría de las veces; algunas veces; solo unas pocas veces y nunca. La puntuación del cuestionario está dada por una escala de tipo Likert, que se asocia a cada pregunta, con un máximo de 4 puntos por pregunta, donde una mayor puntuación indica un mayor riesgo. A partir de ello, se estimaron los puntajes de cada dimensión y subdimensión por terciles, con el objeto de establecer rangos: Bajo (nivel de exposición más favorable para la salud), Medio (nivel intermedio) y Alto (nivel de exposición más desfavorable para la salud).

\section{Variables de estudio}

Se definieron variables referidas a características socio-demográficas de los participantes (edad, sexo, nivel de educación y ocupación) y variables relativas a las 5 dimensiones de estudio, las que se definen seguidamente.

Exigencias psicológicas: Esta dimensión tiene dos componentes: el cuantitativo y el tipo de tarea. Desde el punto de vista cuantitativo se refieren al volumen de trabajo en relación al tiempo disponible para hacerlo, en tanto que el tipo de tarea implica exposiciones distintas en función de si se trabaja con y para personas (predominante en el sector salud), con equipamiento tecnológico, etc. En función de esto se definen las exigencias psicológicas de tipo emocional y de tipo cognitivo. ${ }^{14,15}$

Trabajo activo y desarrollo de habilidades: Refleja la autonomía en el día a día y las oportunidades de aplicar y adquirir conocimientos y habilidades. Da cuenta del grado de influencia que el trabajador posee sobre su trabajo, especialmente en el control de los tiempos, y con las posibilidades de desarrollo de habilidades y aprendizajes que le brinda la organización. ${ }^{16}$

Apoyo social y calidad de liderazgo: Valora las relaciones interpersonales en el ámbito de trabajo, las que afectan la salud de diversas formas. ${ }^{17}$ El apoyo social representa el aspecto funcional de estas relaciones (recibir la ayuda adecuada para trabajar de superiores y compañeros/as), mientras que el sentimiento de grupo representa su componente emocional.

Compensaciones: Indaga sobre el grado de seguridad del trabajador sobre la continuidad de su relación laboral y sobre el tipo de tareas que debe realizar, y la estima que percibe en el ámbito laboral (reconocimiento, apoyo adecuado y trato justo). Está basada en el modelo esfuerzo-recompensa, y parte del supuesto que la relación entre un esfuerzo elevado y un bajo nivel de recompensas a largo plazo representa un riesgo para la salud. Por compensaciones del trabajo, se ha postulado el control de estatus, la estima y el salario. ${ }^{18}$ 
Tabla 1. Composición del Cuestionario ISTAS- SUCESO (versión breve).

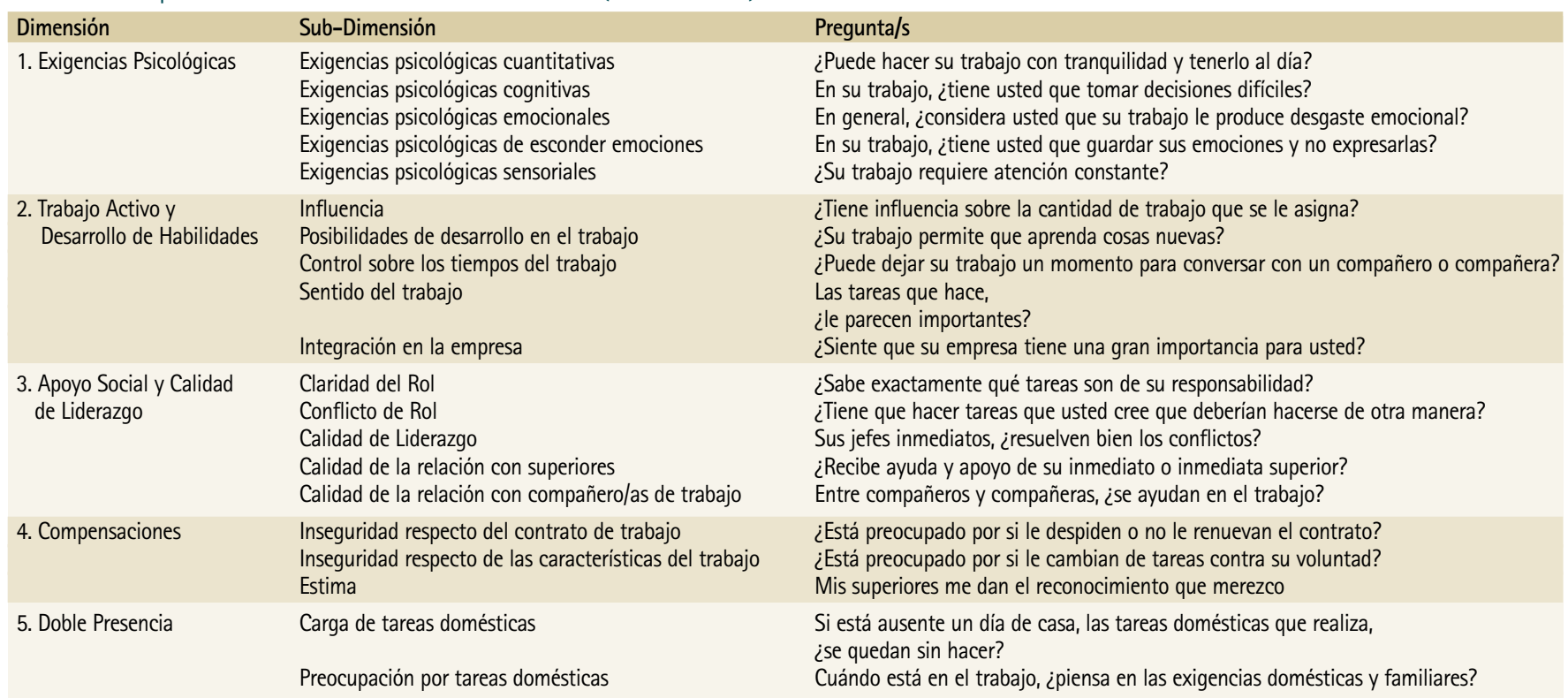

Fuente: elaboración propia en base a la presentación del SUCESO-ISTAS 21 y al manual de uso de la versión breve del mismo instrumento.

Doble presencia: Valora la existencia de exigencias sincrónicas y simultáneas, entre el ámbito laboral y del ámbito doméstico-familiar, considerándose que la necesidad de responder a las demandas del trabajo asalariado y del trabajo doméstico-familiar puede afectar negativamente la salud. La realización del trabajo asalariado y del trabajado doméstico-familiar puede aumentar las demandas y horas de trabajo (doble exposición o doble trabajo). ${ }^{9}$ Además, la necesidad de compaginar ambos trabajos plantea también un conflicto de tiempos, puesto que ambos forman parte de la misma realidad social, principalmente para las mujeres, y presentan interferencias frecuentes en el tiempo y momento que es necesario responder a las demandas de ambos espacios.

\section{Análisis estadístico}

Los datos surgidos de las encuestas fueron capturados en una base de datos para su procesamiento y análisis posterior. Para ello se utilizó el programa SPSS en su versión 20.0 para Windows, en el que se realizó el análisis estadístico descriptivo de los datos con el objeto de explorar el comportamiento de las dimensiones estudiadas. Las respuestas fueron clasificadas en nivel de riesgo Alto (exposición psicosocial más desfavorable para la salud), nivel de riesgo Medio (exposición psicosocial intermedia) y nivel de riesgo Bajo (exposición psicosocial más favorable para la salud). En el análisis estadístico se calcularon medidas de frecuencias relativas (proporciones) y el test de chi cuadrado ( $p$ $<0,05$ ), con un intervalo de confianza (IC) del 95\%.

\section{RESULTADOS}

\section{Características socio-demográficas de los participantes en el estudio}

De las 844 encuestas válidas, 453 (54\%) perteneció a trabajadores que se desempeñan en establecimientos de la ciudad capital y 391 (46\%) en hospitales del interior provincial. El 73\% de las encuestadas fue mujer y el 64\% tenía entre 18 y 45 años. Esta variable tuvo una distribución Normal, coincidiendo la media con la mediana en 41 años. Al respecto de la ocupación del personal encuestado, 54\% fue enfermeros/as, 26\% médicos/as y 20\% perteneció al grupo otros integrantes del equipo de salud (bioquímicos, psicólogos, odontólogos, farmacéuticos, licenciados en Kinesiología y Fisioterapia, licenciados en Fonoaudiología, licenciados en Trabajo Social, técnicos universitarios en Radiología, Laboratorio, Hemoterapia). Por último, en relación al nivel de instrucción, el 58\% de los trabajadores encuestados es universitario, y casi el 8\% tiene un título de postgrado universitario (especialista, magíster o doctorado) de manera que si se suman estos dos grupos se concluye que más del 60\% de los encuestados tiene un alto nivel de instrucción. En la Tabla 2 se exponen los resultados que describen la composición de los trabajadores estudiados.

\section{Tabla 2.}

Características socio-demográficas de los trabajadores encuestados.

\begin{tabular}{llcc} 
Variable & & N & $\%$ \\
Sexo & Femenino & 616 & 73 \\
& Masculino & 228 & 27 \\
Edad & 18 a 45 años & 542 & 64 \\
& 46 o + años & 302 & 35 \\
Nivel de instrucción & Terciario & 291 & 34 \\
& Universitario & 487 & 58 \\
Ocupación & Especialista/Magister/Doctor & 66 & 8 \\
& Médicos/as & 217 & 26 \\
& Enfermeras/os & 456 & 54 \\
\multirow{2}{*}{ Zona del hospital donde trabaja } & Otros integrantes del equipo de salud & 171 & 20 \\
& Capital & 453 & 54 \\
& Interior & 391 & 46
\end{tabular}

\section{Frecuencia de exposición a los riesgos psicosociales en el} ámbito hospitalario

Tal como se observa en la Figura 1, las situaciones más desfavorables (riesgo Alto) se encontraron en las dimensiones: Exigencias psicológicas (57,7\%), apoyo social y calidad de liderazgo $(56,2 \%)$ 
Figura 1.

Distribución de frecuencias del nivel de riesgo según dimensión.

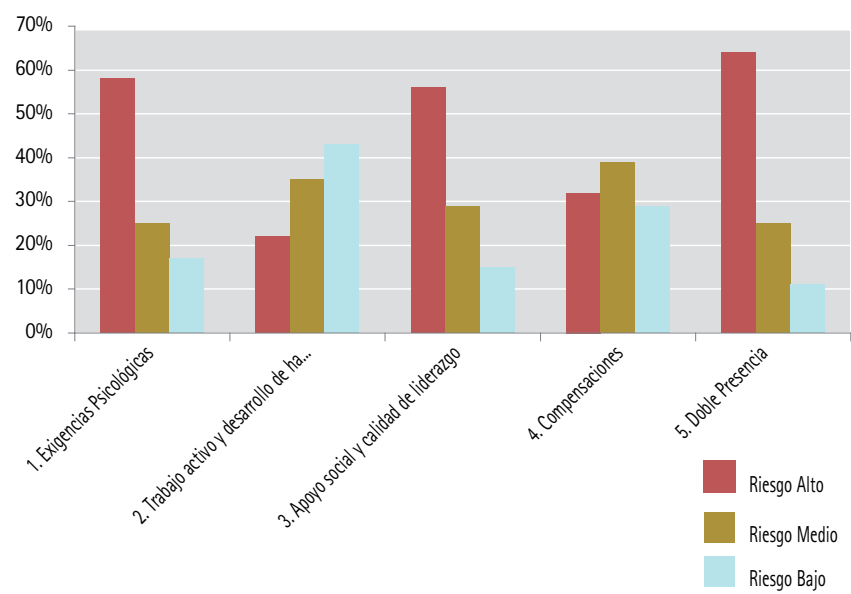

y doble presencia (64\%). El riesgo Medio predominó en la dimensión compensaciones (39\%) y el riesgo Bajo, es decir, donde predomina una situación más favorable, se observó en la dimensión Trabajo activo y desarrollo de habilidades (42,9\%).

$\mathrm{Al}$ examinar las frecuencias de nivel de riesgo Alto en cada dimensión según el tipo de profesión del trabajador se halló que en las dimensiones: Exigencias psicológicas y de Apoyo social y calidad de liderazgo este nivel de riesgo fue más frecuente entre el personal médico en relación al de enfermería y a otros profesionales, con diferencias ( $\mathrm{p}$ $<0,001)$ en el caso de la dimensión de Exigencias psicológicas. En la dimensión Compensaciones el riesgo alto fue más frecuente entre el personal de enfermería, en tanto que las dimensiones de Trabajo activo y posibilidades de desarrollo y Doble presencia resultó más frecuente para otros profesionales (ver Figura 2).

Figura 2.

Distribución de frecuencias de riesgo Alto en cada dimensión, según profesión/ocupación del trabajador.

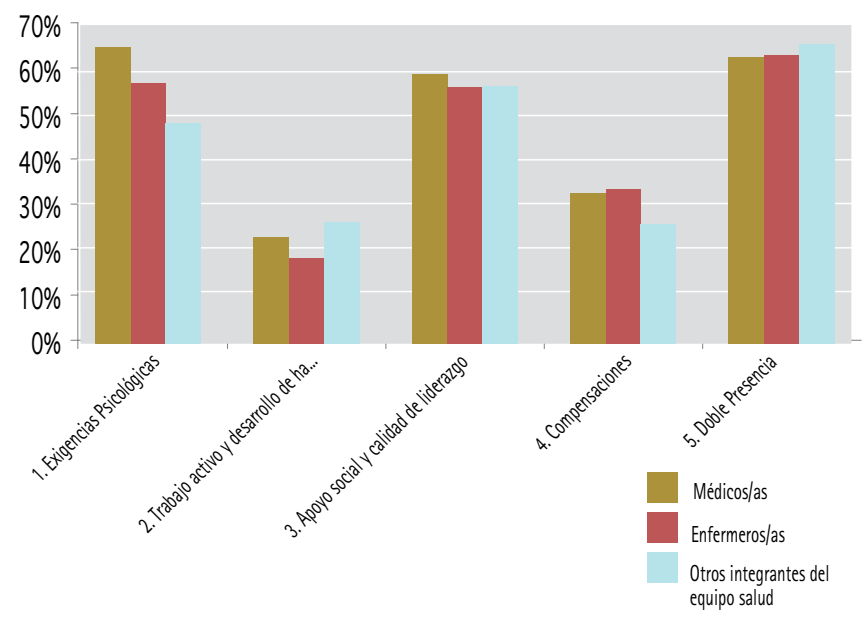

En cuanto a los niveles de riesgo Alto en cada dimensión según el sexo del trabajador, se encontró que las mujeres presentan una mayor proporción de riesgo Alto en las dimensiones de Exigencias psicológicas; Apoyo social y calidad de liderazgo; Compensaciones y en la de Doble presencia. En esta última dimensión se hallaron diferencias $(p<0,001)$. En tanto que los varones presentaron más frecuentemente riesgo Alto en la dimensión de Trabajo activo y posibilidades de desarrollo (ver Figura 3).
Figura 3.

Distribución de frecuencias de riesgo Alto en cada dimensión, según sexo del trabajador.

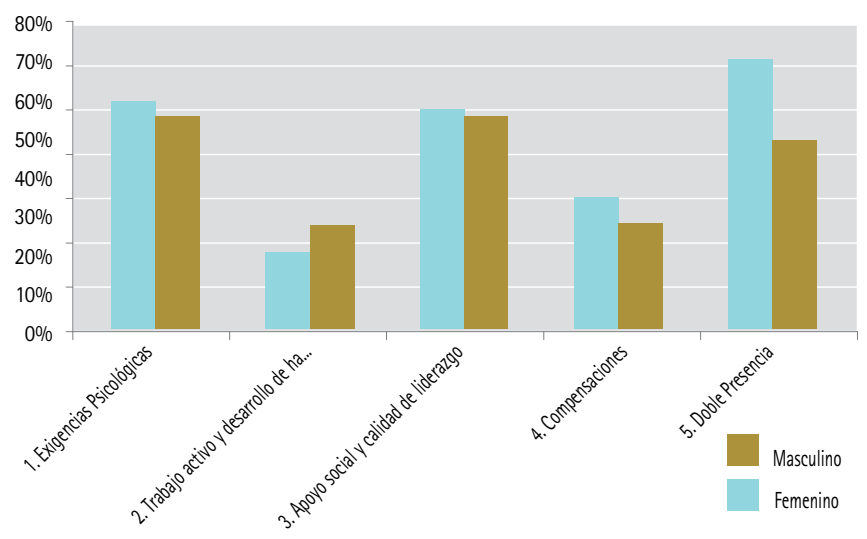

Como puede observarse en la Figura 4, en relación a la antigüedad del personal se halló que los integrantes del equipo de salud con antigüedad intermedia (de 5 a 10 años) presentan mayor frecuencia de riesgo Alto en las dimensiones de Trabajo activo y posibilidades de desarrollo; Apoyo social y calidad de liderazgo y en la de Doble presencia. Los de menor antigüedad tienen mayor frecuencia en la dimensión de Exigencia psicológica. Respecto de la dimensión de Trabajo activo y posibilidades de desarrollo se encontraron diferencias $(p<0,001)$ en la frecuencia de riesgo Alto.

\section{Figura 4.}

Distribución de frecuencias de riesgo Alto en cada dimensión, según antigüedad del trabajador.

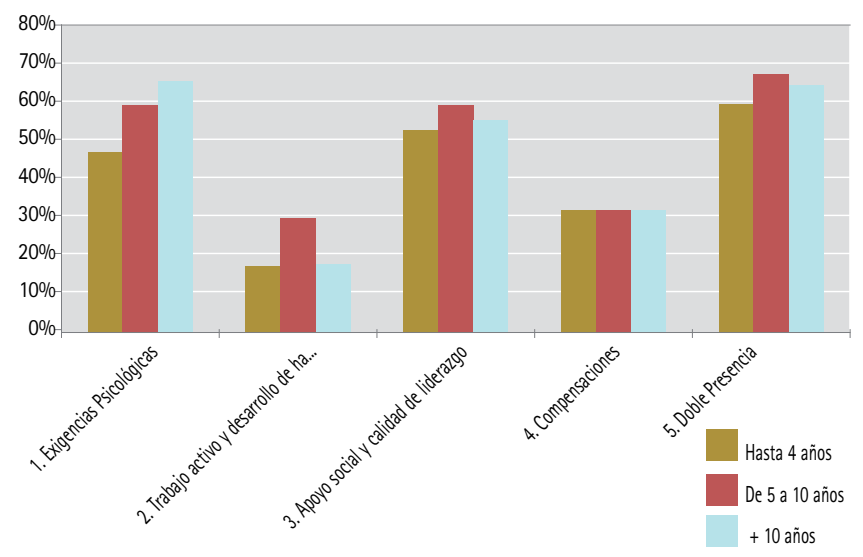

\section{Nivel de riesgo en cada una de las dimensiones y subdi- mensiones estudiadas}

Dimensión 1: Exigencias psicológicas

Predominaron las valoraciones desfavorables, registrándose un $57,7 \%$ de riesgo Alto, un 25,2\% de riesgo Medio y un 17,1\% de riesgo Bajo.

La sub-dimensión relacionada con las exigencias sensoriales fue la que registró niveles más altos de riesgo. Así, el 94\% de los encuestados consideró que su trabajo requiere atención constante siempre o la mayoría de las veces, algunas veces el 3,6\% y solo el 2,4\% eligió las opciones solo una pocas veces o nunca.

Respecto de las exigencias cuantitativas, el 67,8\% de los profesionales encuestados mencionó poder hacer su trabajo con tranquilidad 
y tenerlo al día siempre o la mayoría de las veces; el 23,9\% algunas veces y el 8,3\% solo unas pocas veces o nunca.

A nivel de exigencias psicológicas cognitivas, dijeron tener que tomar decisiones difíciles en su trabajo, siempre o la mayoría de las veces el 31,9\% de los encuestados, algunas veces el 40\%, solo unas pocas veces o nunca el $28,1 \%$.

Cuando se les consultó sobre si en general consideraban que su trabajo les generaba desgaste emocional (exigencia emocional), el $49,8 \%$ señaló que siempre o la mayoría de las veces, el 29\% refirió que algunas veces, en tanto que el 21,2\% de los profesionales encuestados seleccionó solo unas pocas veces o nunca.

En relación a si en su trabajo debe guardar sus emociones y no expresarlas, el 49,6\% seleccionó siempre o la mayoría de las veces, el $28,3 \%$ refirió que algunas veces, el 22,1\% eligió nunca o solo unas pocas veces.

Para la dimensión Exigencias psicológicas se encontraron diferencias $(p<0,05)$ en la situación de Riesgo Psicosocial Alto en relación al nivel educativo de los encuestados, que resultó ser más frecuente entre los profesionales con nivel de instrucción universitario, que entre el personal con menor nivel de instrucción, y entre médicos/as respecto del resto de las profesiones/ocupaciones.

\section{Dimensión 2: Trabajo activo y desarrollo de habilidades}

En esta segunda dimensión, se encontró la situación más favorable de los cinco grupos, predominando un nivel de riesgo Bajo $(42,9 \%)$, seguido por el riesgo Medio $(35,4 \%)$ y una menor proporción de los profesionales está en riesgo Alto (21,7\%).

En la subdimensión que valora el grado de integración a su hospital, al indagar sobre la importancia que tiene para el encuestado "su institución" el 69,7\% respondió siempre o la mayoría de las veces, el 18\% refirió que algunas veces y el 12,3\% nunca o solo unas pocas veces.

En cuanto al sentido del trabajo, el 93,4\% mencionó que siempre o la mayoria de las veces las tareas que realiza le parecen importantes, el 5,2\% señaló que algunas veces y solo el 1,5\% nunca o solo unas pocas veces.

Respecto de la influencia sobre la cantidad de tareas que se le asigna el 40\% de los encuestados dijo que solo unas pocas veces o nunca, el 35\% respondió siempre o la mayoría de las veces, y el 25\% algunas veces. Esta sub-dimensión resultó la más desfavorable dentro de la dimensión 2.

En relación al aprendizaje y posibilidades de desarrollo en el ámbito laboral, al ser consultados sobre si su trabajo les permite aprender cosas nuevas, los encuestados respondieron: siempre o la mayoría de las veces el 76,7\%, algunas veces el 16,4\%, y el 6,9\% solo unas pocas veces o nunca.

En lo relativo al grado de control sobre los tiempos en el trabajo, los encuestados consultados sobre la posibilidad de dejar su trabajo un momento para conversar con un compañero/a, la mayoría $(48,7 \%)$ eligió la opción algunas veces, el 35,3\% mencionó que siempre o la mayoría de las veces, y el 16\% eligió solo unas pocas veces o nunca.

\section{Dimensión 3: Apoyo social y Calidad de liderazgo}

Aquí resultó más frecuente el nivel de riesgo Alto $(56,2 \%)$, encontrándose un nivel de riesgo medio en el 28,8\% de los casos y un 15\% de riesgo bajo.

En relación al conflicto de rol, el 29,5\% de los profesionales encuestados respondió que siempre o la mayoría de las veces tiene que hacer tareas que cree deberian hacerse de otra manera, el 45,6\% algunas veces, y $24,9 \%$ mencionó que nunca o solo unas pocas veces.
Respecto de la claridad del rol, los trabajadores encuestados dijeron saber exactamente qué tareas son de su responsabilidad la mayoria de las veces o siempre en un 91,7\% de los casos, en tanto que el 8,3\% expresó que eso ocurre algunas veces, solo unas pocas veces o nunca. En cuanto a la calidad del liderazgo, los encuestados valoraron que los jefes inmediatos resuelven bien los conflictos, solo unas pocas veces o nunca en un 25,2\% de los casos, el 28,4\% respondió algunas veces, y que lo hacen la mayoría de las veces o siempre el $46,4 \%$. La calidad de la relación con superiores presenta también un predominio de respuestas desfavorables dado que el 28,7\% respondió que nunca o solo unas pocas veces recibe ayuda o apoyo del inmediato superior, el 24,6\% que algunas veces, y que lo recibe la mayoría de las veces o siempre el $46,7 \%$.

En tanto que, se hallaron respuestas predominantemente favorables en relación a la ayuda entre compañeros de trabajo, así un 72,2\% refirió que eso ocurre siempre y la mayoría de las veces, que algunas veces el 19,9\% y que solo unas pocas veces o nunca el 7,9\%.

\section{Dimensión 4: Compensaciones}

Para esta dimensión la mayoría de los trabajadores encuestados (39\%) se encuentra en situación de riesgo psicosocial Medio, un 32\% está en riesgo Alto y el 29\% se encuentra en la categoría Bajo riesgo.

En relación a la inseguridad respecto del contrato de trabajo, el 19,3\% de los encuestados mencionó estar siempre o la mayoría de las veces preocupado por si le despiden o no le renuevan el contrato, el $7,5 \%$ escogió la opción algunas veces y el 73,2\% solo unas pocas veces o nunca.

Respecto de la preocupación por el cambio de tarea contra su voluntad, un 21,4\% dijo estar siempre o la mayoría de las veces preocupado, el 15,6\% algunas veces, y el 62,9\% mencionó solo unas pocas veces o nunca.

En cuanto a si recibe el reconocimiento que merece, el 39\% señaló que solo unas pocas veces o nunca, el 27\% algunas veces, y el 34\% mencionó que siempre o la mayoría de las veces ocurre eso.

\section{Dimensión 5: Doble presencia}

Resultó la más desfavorable de las cinco de dimensiones estudiadas, encontrándose un nivel de riesgo psicosocial Alto del 64\%, Medio del 25,4\% y Bajo del 10,6\%.

En relación a la carga de tareas domésticas, el 43,1\% respondió que siempre o la mayoría de las veces que está ausente un día de casa, las tareas domésticas que realiza quedan sin hacer, el 26\% respondió algunas veces, y el 31\% que nunca o solo unas pocas veces.

Respecto de la preocupación por las tareas domésticas, el 29,2\% manifestó que siempre o la mayoría de las veces cuando está en el trabajo piensa en las exigencias domésticas y familiares, el 36\% respondió algunas veces, y el 34,8\% respondió que sólo unas pocas veces o nunca.

Para esta dimensión se hallaron diferencias $(p<0,01)$ entre sexos, siendo más frecuente el riesgo Alto en mujeres que en varones.

\section{DISCUSION}

Los cambios en el mundo del trabajo ocurridos en las últimas décadas han repercutido sobre las condiciones de trabajo y los riesgos laborales a los que se exponen los trabajadores, en particular en el sector salud, donde los riesgos psicosociales son reconocidos más frecuentemente y su estudio va adquiriendo mayor interés debido a las implicancias que estos tienen. 
En la presente investigación se halló que en los trabajadores de la salud predominan riesgos psicosociales Altos en tres de las cinco dimensiones estudiadas: Exigencias psicológicas; Apoyo social y calidad de liderazgo; y Doble presencia, en tanto que otros estudios efectuados sobre el personal de salud, donde se aplicó una metodología similar reveló que en la totalidad de las dimensiones predominaba el riesgo Alto. ${ }^{20}$

$\mathrm{Al}$ respecto de las Exigencias psicológicas, estas resultaron más elevadas en el personal de mayor nivel educativo, en el grupo de médicos y en los de más antigüedad laboral, situación que posiblemente puede estar relacionada con el mayor grado de responsabilidad y atención que requieren sus actividades. En este sentido, cabe señalarse que la subdimensión de exigencias sensoriales, que valora el grado de atención que requieren las tareas, fue la que registró el mayor nivel de riesgo dentro de esta dimensión. Estos hallazgos tienen similitud con los comunicados en un estudio realizado en Michelín, España, en médicos de un hospital, donde los encuestados identifican riesgos asociados a exigencias psicológicas en cifras mayores al 80\% y la percepción de estos aumenta con la antigüedad en ese empleo. ${ }^{21}$ En el mismo sentido, una investigación cualitativa sobre médicos especialistas de hospitales públicos de Valencia, España, también pone de manifiesto que este personal expresa un alto número de factores de riesgo psicosocial relacionados con las exigencias psicológicas, algunos vinculados específicamente con las características de la profesión médica (contacto con el sufrimiento y con la muerte, sentirse responsable de vidas humanas, incertidumbre ante el diagnóstico y el tratamiento) y otros con la organización del trabajo (sobrecarga de trabajo y falta de personal). ${ }^{22}$

En la dimensión de Apoyo social y calidad del liderazgo, los niveles de riesgo más altos se registraron en el personal médico y en el de sexo femenino, los que podrían estar vinculados con los estilos de conducción y liderazgo existentes en el ámbito hospitalario. En tal sentido, la relación entre estilos de conducción y riesgos psicosociales ha sido documentada en diversas investigaciones, encontrándose que estilos de dirección con altos niveles de exigencia y coerción afectan de manera importante la percepción de clima organizacional ${ }^{23}$ y estilos que fomentan roles confusos y que no favorecen la autonomía en la toma de decisiones afectan la salud del trabajador. ${ }^{24}$ Asimismo, Evangelina Prince menciona que "Las oportunidades para el ejercicio del liderazgo por las mujeres siguen siendo restringidas, no sólo por los factores del marco cultural general excluyente, sino por culturas políticas, administrativas y organizacionales que reproducen los valores androcéntricos de la cultura general y que se reflejan fielmente en leyes, normas y procedimientos institucionales y que son efectivas barreras a la igualdad". ${ }^{25}$ Otro aspecto que podría explicar el nivel de riesgo de esta dimensión son las deficiencias en la estandarización de las tareas, que en algunos casos pueden resultar demasiado rígidas y en otros casi inexistentes, lo que se refleja en la elevada proporción de encuestados que refiere conflictos de rol. Así, las situaciones de ambigüedad y de conflicto de rol en el trabajo, repercuten negativamente en el bienestar psicológico del trabajador y se las considera como fuentes de tensión para la persona que así las vive, contribuyendo al estrés en el trabajo. ${ }^{26}$ Estos hallazgos se corresponden con los publicados en un estudio realizado en centros de Atención Primaria de Salud en una comuna de Santiago, Chile, donde los resultados para estrés emocional mostraron que conflicto de rol y apoyo de pares se mantuvieron significativamente asociadas con sintomas de estrés emocional, a la vez que mostraron que las mujeres presentan puntajes significativamente mayores en las escalas de sintomas de estrés emocional y estrés físico. ${ }^{27}$

La Doble presencia resultó la dimensión con mayor proporción de riesgo alto, con una amplia desventaja para las mujeres. Esta situación podría relacionarse con lo postulado por Pilar Carrasquer quien señaló que, desde el punto de vista del empleo femenino, la modernidad resulta un mito, ya que esta modernidad está vista solo en una de sus caras, la de la esfera pública,y, en concreto, la del trabajo remunerado, sin considerar que esa propia modernidad se ha construido, además, sobre el trabajo cotidiano no reconocido de las mujeres adultas, esto es sobre el trabajo doméstico familiar socialmente adjudicado al género femenino. ${ }^{28}$ Según datos disponibles a nivel europeo, las mujeres de 20 a 74 años dedican más tiempo que los hombres al trabajo doméstico, una cifra que crece en los casos de Italia, Estonia, Eslovaquia, Hungría y España alrededor de cinco o más horas por día. Mientras que las mujeres de Suiza, Noruega y Finlandia son las que menos tiempo dedican, por debajo de las cuatro horas diarias. En contraste con eso, los hombres dedican más tiempo al trabajo remunerado y a los estudios que a las tareas domésticas $\mathrm{y}$, en todos los países, las mujeres tienen menos tiempo libre que los hombres. ${ }^{29}$

Además de esta diferencia entre sexos, es notorio lo elevado del porcentaje global de riesgo Alto para Doble presencia encontrado en esta investigación, especialmente en personal de antigüedad laboral intermedia (5 a 10 años), que posiblemente coincida con un momento del ciclo vital relacionado con el crecimiento familiar en aquellas familias o parejas más modernas o que no establecen los roles de proveedor para el hombre y reproductora para la mujer de manera tan radical. Los datos obtenidos coinciden con lo hallado en una investigación realizada en el hospital de la Facultad de Medicina de Witten, Alemania, donde se señala que la incompatibilidad entre trabajo y familia se considera como una de las principales razones de renuncia de los médicos del hospital, que deciden emplearse en trabajos no relacionados con la clínica o mudarse a otros paises. ${ }^{30}$

En la dimensión Compensaciones, si bien predominó la frecuencia de riesgo Medio, el 32\% de los encuestados está en riesgo Alto para esta dimensión, con mayor frecuencia entre mujeres y personal de enfermería. Si se considera que esta dimensión evalúa principalmente la seguridad en el empleo, cabría relacionar esto con la proporción de personal con vínculos laborales no permanentes, estimado en el 36\% en un estudio recientemente realizado sobre la misma población de trabajadores. ${ }^{31}$ Esta situación podría tener serias implicancias sobre la salud de los trabajadores dado que existen diversos estudios que señalan que la inseguridad en el empleo es un importante estresor psicológico y que la amenaza de quedarse sin empleo o de perder el control de las actividades laborales puede generar angustia mental ${ }^{32}$ y contribuir a la aparición de problemas psiquiátricos. Un estudio de cohortes efectuado en Suecia reveló que el empleo temporal puede tener efectos adversos sobre la percepción de la salud y la salud psicológica ${ }^{34}$, en tanto que una investigación exploratoria sobre la población asalariada en España halló que las buenas exposiciones psicosociales están relacionados con los métodos de trabajo participativos, con jefes de modales no autoritarios y no agresivos, un contrato de trabajo permanente y no estar amenazado con el despido. ${ }^{35}$ Por otra parte, la inseguridad laboral ha sido asociada a mayor incidencia de enfermedad coronaria $^{36}$ y a mayor dependencia del alcohol. ${ }^{37}$ Además, merece especial atención la baja proporción de trabaja- 
dores, hallada en nuestro estudio, que percibe que su tarea recibe el reconocimiento esperado, toda vez que este ejerce una influencia decisiva en el desempeño laboral y, por lo tanto, en la calidad del servicio de salud.

En lo que refiere a la dimensión Trabajo activo y posibilidades de desarrollo, esta fue la única que presentó un riesgo Bajo, lo que podría relacionarse con la autonomía que poseen los profesionales para realizar sus tareas, las posibilidades que ofrecen los hospitales públicos para la formación y el aprendizaje continuo, y con la relevancia social que implica el trabajo en este tipo de instituciones. Esto resulta de gran interés dado que la autonomía para el ejercicio de las actividades asistenciales y las posibilidades de realización profesional están asociadas a mayor bienestar psicológico. ${ }^{38}$ Asimismo, esta dimensión fue la única donde los niveles de riesgo alto predominaron en los varones, lo que supondría que estos valoran menos que las mujeres las posibilidades de desarrollo profesional que les ofrece el hospital público. Estos hallazgos guardan similitud con los de una investigación efectuada en Chile en trabajadores de una agencia estatal, donde también se encontraron niveles de riesgos más elevados para esta dimensión en el sexo masculino. ${ }^{39}$

Como conclusión puede señalarse que esta investigación posibilitó determinar el nivel de riesgo psicosocial en el trabajo a que están expuestos los integrantes de los equipos de salud de hospitales dependientes del Ministerio de Salud de la provincia de Córdoba, identificándose aquellos riesgos más frecuentemente percibidos por estos trabajadores.

En función de esto, y entendiendo que las condiciones laborales de exposición crónica a riesgos psicosociales van más allá de ser un problema individual, para constituirse en un problema de salud pública cuando su frecuencia es elevada, resulta de interés profundizar en investigaciones que indaguen sobre la situación de los factores psicosociales en estas instituciones sanitarias.

Además, y dada la evidencia científica disponible en relación al deterioro que provoca en la salud psicofísica la exposición crónica a riesgos psicosociales en el ámbito laboral, se considera necesario orientar más efectivamente acciones y normativas de promoción de ambientes de trabajo más saludables y de prevención de estos riesgos, en particular los vinculados a las dimensiones de Exigencias psicológicas, Apoyo social y calidad de liderazgo, y Doble presencia, que fueron aquellas donde predominaron los niveles de riesgo Alto.

\section{Institución donde se realizó la investigación}

- Centro de Investigación y Formación en Gestión de Servicios de Salud y Trabajo (CeGeSST), Escuela de Salud Pública, Facultad de Ciencias Médicas, Universidad Nacional de Córdoba.

- Cátedra de Medicina Preventiva y Social, Facultad de Ciencias Médicas, Universidad Nacional de Córdoba.

\section{Fuentes de financiamiento}

-Beca "Carrillo-Oñativia", Convocatoria 2011. Comisión Nacional Salud Investiga, Ministerio de Salud de la Nación, Argentina.

- Subsidio de la Secretaría de Ciencia y Tecnología de Universidad Nacional de Córdoba. Convocatoria a Proyectos y Programas de Investigación y Desarrollo 2012-2013.

\section{REFERENCIAS}

1. Acevedo G, Farías A, Sánchez J. Trabajar en el sector salud. Factores Influyentes en las condiciones y medioambiente de trabajo. Córdoba, Argentina: Universidad Nacional de Córdoba; 2011. 125 p.

2. Gil Monte PR. Riesgos psicosociales en el trabajo y salud ocupacional. Rev Perú Med Exp Salud Pública. 2012; 29(2):237-41.

3. Gil Monte PR. Algunas razones para considerar los riesgos psicosociales en el trabajo y sus consecuencias en la salud pública. Rev Esp Salud Pública. 2009; 83(2):169-73.

4. Muñiz Saborido JR. Factores Psicosociales. En: Gestal JJ, Acevedo G. Salud Laboral, Prevención de Riesgos en el Trabajo, Aplicación al Sector Salud en la República Argentina. Santiago de Compostela: Universidad de Santiago de Compostela; 2009. pp. 407-17.

5. Dollard MF, LaMontagne AD, Caulfield N, Blewett V, Shaw A. Job stress in the Australian and international health and community services sector: a review of the literature. Int J Stress Manag. 2007; 14:417-45.
6. Eller NH, Netterstrøm B, Gyntelberg F, Kristensen TS, Nielsen F, Steptoe A, Theorell T. Work-related psychosocial factors and the development of ischemic heart disease: a systematic review. Cardiol Rev. 2009;17(2):83-97.

7. Melamed S, Shirom A, Toker S, Berliner S, Shapira I. Burnout and risk of cardiovascular disease: evidence, possible causal paths, and promising research directions. Psychol Bull. 2006; 132:327-53.

8. Warren N. Psychosocial and work organizational risk factors for work related musculoesqueletal disorders. En: Karwoswki W. International Encyclopedia of Ergonomics and Human Factors. Oxford: CRC Press; 2010. pp. 1299-1302.

9. Grau A, Flichtentrei D, Suñer R, Prats M, Braga F. El sindrome de burnout en profesionales sanitarios hispanoamericanos y españoles. Influencia de factores personales, profesionales y nacionales. Rev Esp Salud Pública. 2009; 8:215-230. 
10. Parent-Thirion A, Fernández E, Hurley J, Vermeylen G. Fourth European Working Conditions Survey [on line]. Luxembourg: European Communities Press; 2007. 139 p.[Consultado 24 ago 2013]. Disponible en: http://www.eurofound.europa. eu/pubdocs/2006/98/en/2/ef0698en.pdf

11. NIOSH. Worker Health Chartbook 2004[on line]. Cincinnati, OH: NIOSH; 2004. 34 p. [Consultado 24 ago 2013]. Disponible en: http://www.cdc.gov/niosh/docs/ chartbook/

12. España. Instituto Nacional de Seguridad e Higiene en el Trabajo. VI Encuesta de Condiciones de Trabajo. Madrid: MTAS; 2008. P. 183.

13. Chile. Superintendencia de la Seguridad Social. Cuestionario de Evaluación de Riesgos Psicosociales en el Trabajo SUSESO-ISTAS 21. Santiago de Chile: SISS; 2009. p 33.

14. Dormann C, Zapf D. Customer-related social stressors and burnout. J Occup Health Psychol. 2004; 9(1):61-82.

15. Vanroelen C, Levecque $K$, Moors G, Gadeyne $S$, Louckx F. The structuring of occupational stressors in a Post-Fordist work environment. Moving beyond traditional accounts of demand, control and support. Soc Sci Med. 2009; 68(6): 1082-1090.

16. Ala Mursula L, Vahtera J, Linna A, Pentti J, Kivimäki M. Employee worktime control moderates the effect of job strain and effort-reward imbalance on sickness absence: the 10-town study. J Epidemiol Community Health. 2005; 59(10):851-7.

17. Stoetzer U, Ahlberg G, Johansson G, Bergman P, Hallesten L, Forsell Y, Lundberg I. Problematic Interpersonal Relationships at Work and Depressive: A Swedish Prospective Cohort Study. J Occup Health. 2009; 51:144-151.

18. Siegrist J. Adverse health effects of high-effort/low reward conditions. J Occup Psychol. 1996; 1: 27-41.

19. Chandola $T$, Martikainen $P$, Bartley M, Lahelma E, Marmot M, Michikazu S, et al. Does conflict between home and work explain the effect of multiple roles on mental health? A comparative study of Finland, Japan, and the UK. Int J Epidemiol. 2004; 33(4):884-893.

20. Marin-Lluch $P$, Garcia-Domingo $C$, Muñoz-Fernández JL, Rabanaque-Mallent G. Detección de riesgos psicosociales en profesionales sanitarios de un área de salud. Atención Primaria. 2006; 35 (5):302-303.

21. Prieto, Gamo y Figueroa. Percepción de los riesgos de su puesto de trabajo de los médicos internos residentes de un hospital secundario. Educ Med. 2011;14(3):163-170.

22. Escribà Agüir V, Bernabé-Muñoz Y. Exigencias laborales psicológicas percibidas por médicos especialistas hospitalarios. Gaceta Sanitaria 2.002; 16(6):487-496.

23. Bloch S, Whiteley P. Complete Leadership: A practical guide for developing your leadership talents. London: Pearson Education; 2003.

24. Contreras F, Barbosa D, Juárez F, Uribe AF, Mejia C. Estilos de liderazgo, clima organizacional y riesgos psicosociales en entidades del sector salud. Un estudio comparativo. Acta Colombiana de Psicología. 2009; 12(2):13-26.

25. Prince García E. Las mujeres en las estructuras de Poder y de Toma de Decisiones (Documento Oficial). Reunión de Consulta de las Delegadas Comisión Interamericana de Mujeres de la Organización de Estados Americanos, Washington, feb.1998. Washington: OEA; 1998.

26. España. Ministerio de Trabajo y Asuntos Sociales. Instituto Nacional de Seguridad e Higiene en el Trabajo. NTP 388: Ambigüedad y Conflicto de Rol. Madrid: INSHT; 1998.
27. Guic $E$, Mora $P$, Rey $R$, Robles $A$. Estrés organizacional y salud en funcionarios de centros de atención primaria de una comuna de Santiago[en línea]. Rev méd Chile. 2006; 134(4):447-455. [Consultadol 4 sep 2013]. Disponible en:http://www.scielo.cl/scielo.php?script=sci_arttext\&pid=S0034-9887200 $6000400007 \&$ Ing $=$ es

28. Pilar Carrasquer Oto. La doble presencia: el trabajo y el empleo femenino en las sociedades contemporáneas. [Tesis Doctoral]. Barcelona: Universidad Autónoma de Barcelona; 2009.

29. Colom, Sara Moreno. Uso del Tiempo, desigualdades sociales y ciclo de vida. Nota de Investigación. Barcelona: Universidad Autónoma de Barcelona; 2009.

30. Fub I, Nubling $M$, Hasselhorn HM, Schwappach $M$, Rieger M. Working conditions and work-family conflict in German hospital physicians: psychological and organisational predictors and consequences. BMC Public Health [on line]2008; 8:353 [Consultado 8 sep 2013]. Disponible en: http://www. biomedcentral.com/1471-2458/8/353\%20?iframe $=$ true $\&$ width $=$ $100 \%$ \&height $=100 \%$

31. Acevedo G, Farias A, Sánchez J, Astegiano C, Buffa G, Álvarez Loyaute GF, et al. Condiciones y medio ambiente de trabajo en hospitales públicos provinciales de la ciudad de Córdoba. Rev Salud Pública. De próxima aparición 2014.

32. Sirviö A, Ek E, Jokelainen J, Koiranen M, Järvikoski T, Taanila A. Precariousness and discontinuous work history in association with health. Scand J Public Health. 2012; 40(4):360-367.

33. Organización Internacional del Trabajo (0.I.T.). Factores psicosociales y de organización. En: Enciclopedia de salud y seguridad en el trabajo. $3^{\circ}$ ed. Madrid: Ministerio de Trabajo y Asuntos Sociales; 2001.

34. Waenerlund $A$, Virtanen $P$, Hammarström $A$. Is temporary employment related to health status? Analysis of the Northern Swedish Cohort [on line]. Scand J Public Health. 2011; 39(5):533-539. [Consultado 10 septiembre 2013]. Disponible en: $h t t p: / / s j p . s a g e p u b . c o m /$ content/39/5/533.short

35. Llorens $C$, Alós $R$, Cano E, Font A, Jódar P, López V. Psychosocial risk exposures and labour management practices. Scand J Public Health [on line]. 2010;38(3 supl):125-136 [Consultado 10 sept 2013). Disponible en: http:// sjp.sagepub.com/content/38/3_suppl/125.short

36. Virtanen $M$, Nyberg $S$, Batty $P D$, Jokela $M$, Heikkilä $K$, Fransson $E_{1}$ et al. Perceived job insecurity as a risk factor for incident coronary heart disease: systematic review and meta-analysis [on line]. BMJ. 2013;347:f4746 [Consultado 11 sep 2013). Disponible en: http://www.bmj.com/content/347/ bmj.f4746

37. Associations between Temporary Employment, Alcohol Dependence and Cigarette Smoking among Turkish Health Care Workers. Econ Ind Democracy. 2008;29(3):388-405.

38. Paredes $\mathrm{OL}$, Sanabria Ferrand PA. Prevalencia del sindrome de burnout en residentes de especialidades médico quirúrgicas, su relación con el bienestar psicológico y con variables sociodemográficas y laborales. Rev Fac Med. 2008;16(1):25-32.

39. Vera A, Cancino L, Ravanal I, Carrasco C, Contreras G, Arteaga O. Síndrome de Burnout e Inteligencia Emocional: Un Análisis con Enfoque Psicosocial en una Agencia Estatal Chilena [en línea]. Cienc Trab. 2007;9(24):51-54[Consultado 11 sep 2013).Disponible en: http://www. cienciaytrabajo.cl 